\section{§17. Analytic Equilibria of High-Beta Tokamaks with Toroidal and Poloidal Flows and Pressure Anisotropy Associated with Parallel Heat Flux}

Ito, A., Nakajima, N.

Analytic solutions for a reduced set of MHD equilibrium equations for high-beta tokamaks with toroidal and poloidal flow velocities comparable to the poloidal sound velocity as well as with pressure anisotropy associated with parallel heat flux are studied ${ }^{1)}$. The reduced set of equilibrium equations is obtained from the fluid moment equations for collisionless magnetized plasmas by asymptotic expansions with respect to the inverse aspect ratio of a torus for high-beta tokamaks ${ }^{2}$. Its single-fluid limit, where the FLR and two-fluid effects are neglected, is suitable for finding analytic solutions similar to those in the adiabatic pressure case ${ }^{3)}$, which show the modification of both the magnetic structure and the pressure profile due to flow.

From the analytic solution we examine the effect of pressure anisotropy for a fixed poloidal Mach number in the shaded region in Fig. 1 where the equilibrium is regular and the poloidal Mach number lies between the two singular points, which correspond to the phase velocities of sound waves, for all values of the ratio between the parallel and perpendicular pressures for ions, $\alpha_{l}$, as an example. Figure 2 shows the profiles of the parallel and perpendicular pressures for ions and electrons, the total pressure and magnetic flux in the midplane (left), and the distribution of the radial component of the diamagnetic current in a poloidal cross section (right) for the poloidal Mach number 0.45 that are within the shaded region of Fig. 1 and $\alpha_{l}=2.0$ (top) and 0.75 (bottom). The profiles of anisotropic pressures for ions and electrons, the total pressure, and the magnetic flux have different maxima. The diamagnetic current depends on the poloidal Mach number as well as $\alpha_{i}$. If $\alpha_{t}$ is changed from 2.0 to 0.75 , the profiles of pressures are modified and the amplitude of the diamagnetic current is decreased. For comparison, we increase the poloidal Mach number from 0.45 to 1.0 at a fixed $\alpha_{l}$ of 2.0. In this case, the equilibrium moves from the super poloidal sonic region to the sub poloidal sonic one as mentioned above, and the amplitude of the diamagnetic current is decreased, while the profiles of pressure components are not much different. These results come from the fact that decreasing $\alpha_{\imath}$ with the poloidal Mach number fixed in the shaded region moves the equilibrium from the super poloidal sonic region to the sub poloidal sonic one similarly to how increasing the poloidal Mach number at a fixed $\alpha_{t}$ does.

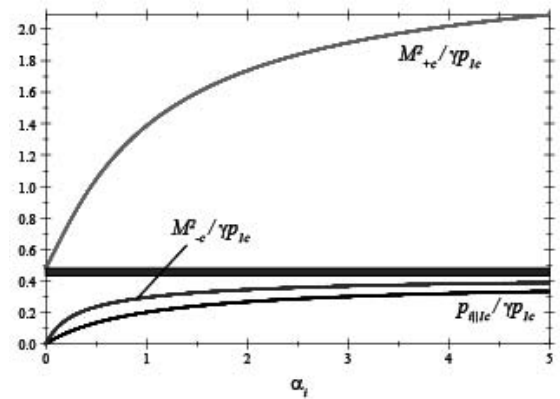

Fig.1 Dependences of poloidal sonic singularity on the pressure anisotropy for ions, $\alpha_{i}$.
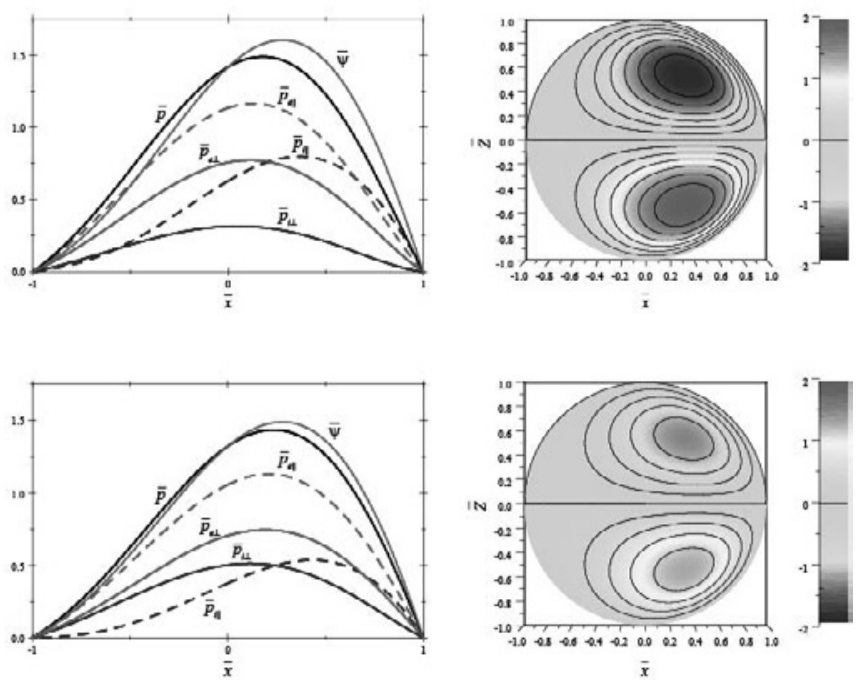

Fig.2 (Left) Profiles of pressures and the magnetic flux in the midplane and (right) the radial component of the diamagnetic current in a poloidal cross section for the poloidal Mach number $0.45, \alpha_{i}=2.0$ (top) and 0.75 (bottom).

1) Ito, A. and Nakajima, N.: J. Phys. Soc. Jpn. 82 (2013) 064502 .

2) Ito, A. and Nakajima, N.: Nucl. Fusion 51 (2011) 123006.

3) Ito, A. and Nakajima, N.: Plasma Phys. Control. Fusion 51 (2009) 035007. 